\title{
Universiteit
}

Leiden

The Netherlands

\section{Excitations in photoactive molecules from quantum Monte Carlo}

Schautz, F.; Buda, F.; Filippi, C.

\section{Citation}

Schautz, F., Buda, F., \& Filippi, C. (2004). Excitations in photoactive molecules from quantum Monte Carlo. Journal Of Chemical Physics, 121(12), 5836. doi:10.1063/1.1777212

Version: $\quad$ Not Applicable (or Unknown)

License: $\quad$ Leiden University Non-exclusive license

Downloaded from: https://hdl.handle.net/1887/67177

Note: To cite this publication please use the final published version (if applicable). 


\section{Excitations in photoactive molecules from quantum Monte Carlo}

Friedemann Schautz, Francesco Buda, and Claudia Filippi

Citation: J. Chem. Phys. 121, 5836 (2004); doi: 10.1063/1.1777212

View online: https://doi.org/10.1063/1.1777212

View Table of Contents: http://aip.scitation.org/toc/jcp/121/12

Published by the American Institute of Physics

\section{Articles you may be interested in}

Energy-consistent pseudopotentials for quantum Monte Carlo calculations

The Journal of Chemical Physics 126, 234105 (2007); 10.1063/1.2741534

Benchmarks for electronically excited states: CASPT2, CC2, CCSD, and CC3

The Journal of Chemical Physics 128, 134110 (2008); 10.1063/1.2889385

Charge-transfer excited states: Seeking a balanced and efficient wave function ansatz in variational Monte Carlo The Journal of Chemical Physics 147, 194101 (2017); 10.1063/1.4998197

Optimized Jastrow-Slater wave functions for ground and excited states: Application to the lowest states of ethene

The Journal of Chemical Physics 120, 10931 (2004); 10.1063/1.1752881

Energy-consistent small-core pseudopotentials for $3 d$-transition metals adapted to quantum Monte Carlo calculations

The Journal of Chemical Physics 129, 164115 (2008); 10.1063/1.2987872

Simple formalism for efficient derivatives and multi-determinant expansions in quantum Monte Carlo

The Journal of Chemical Physics 144, 194105 (2016); 10.1063/1.4948778

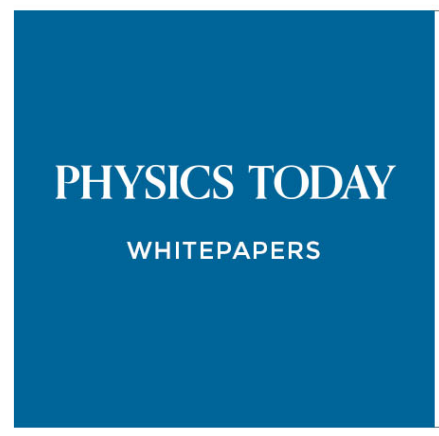

ADVANCED LIGHT CURE ADHESIVES

Take a closer look at what these environmentally friendly adhesive systems can do

\section{READ NOW}

PRESENTED BY

Q8 MASTERBOND 


\title{
Excitations in photoactive molecules from quantum Monte Carlo
}

\author{
Friedemann Schautz \\ Instituut-Lorentz, Universiteit Leiden, Niels Bohrweg 2, 2333 CA Leiden, The Netherlands \\ Francesco Buda \\ Leiden Institute of Chemistry, Gorlaeus Laboratoria, P.O. Box 9502, 2300 RA Leiden, The Netherlands \\ Claudia Filippi \\ Instituut-Lorentz, Universiteit Leiden, Niels Bohrweg 2, 2333 CA Leiden, The Netherlands
}

(Received 21 April 2004; accepted 7 June 2004)

\begin{abstract}
Despite significant advances in electronic structure methods for the treatment of excited states, attaining an accurate description of the photoinduced processes in photoactive biomolecules is proving very difficult. For the prototypical photosensitive molecules, formaldimine, formaldehyde, and a minimal protonated Schiff base model of the retinal chromophore, we investigate the performance of various approaches generally considered promising for the computation of excited potential energy surfaces. We show that quantum Monte Carlo can accurately estimate the excitation energies of the studied systems if one constructs carefully the trial wave function, including in most cases the reoptimization of its determinantal part within quantum Monte Carlo. While time-dependent density functional theory and quantum Monte Carlo are generally in reasonable agreement, they yield a qualitatively different description of the isomerization of the Schiff base model. Finally, we find that the restricted open shell Kohn-Sham method is at variance with quantum Monte Carlo in estimating the lowest-singlet excited state potential energy surface for low-symmetry molecular structures. (c) 2004 American Institute of Physics.
\end{abstract}

[DOI: $10.1063 / 1.1777212$ ]

\section{INTRODUCTION}

The absorption of visible light and its conversion to other forms of energy is at the heart of some of the most fundamental processes in biology. A familiar example of light absorption initiating a biological response is the primary event of vision: light induces a conformational change in rhodopsin, the photoreceptor in the retina, which is followed by a cascade of chemical reactions culminating in the stimulation of the optical nerve. A microscopic understanding of light induced conformational changes in photoactive biomolecules is both important from a fundamental point of view and because of existing and potential applications in biology and biotechnology.

The advances in understanding biological photosystems are so far mainly due to experimental discoveries since theoretical studies are currently hindered by the lack of a theoretical approach which is applicable to realistically large systems while possessing a sufficient degree of reliability. On the one hand, several accurate quantum chemical approaches have been developed for a proper description of excited states but they are only applicable to relatively small systems. For instance, complete active space second-order perturbation theory (CASPT2) ${ }^{1}$ has been employed to investigate the photoisomerization mechanism in simple models of the retinal chromophore of rhodopsin. ${ }^{2-4}$ The approach is able to accurately describe the excited state potential energy surface along the photoisomerization path, but it is limited to relatively small model compounds and a proper description of the important ligand-protein interactions is still computationally prohibitive. On the other hand, density functional theory (DFT) based approaches have a much more favorable scaling with system size than CASPT2 and can therefore be applied to considerably larger molecules. In particular, the restricted open-shell Kohn-Sham method (ROKS) (Refs. 5 and 6) has been recently developed to study the dynamics in low-spin excited states and used to model the full retinal chromophore, including relevant parts of the protein environment. ${ }^{7}$ The resulting excited state potential energy surface along the isomerization coordinate is qualitatively different from the one derived with the CASPT2 method, ${ }^{3}$ though the model systems used in these two works are different and therefore a direct comparison is not possible. Therefore, while the ROKS method is appealing for the low computational cost and for the possibility of performing molecular dynamics in the excited state, its adequateness needs to be further validated. Alternatively, linear response calculations within time-dependent density functional theory (TDDFT) (Ref. 8) often yield accurate excitation energies but fail for instance in describing extended conjugated systems ${ }^{9}$ or proton $\operatorname{transfer}^{10}$ in excited states, that is, systems closely related to photoactive molecules. The capabilities and limitations of TDDFT in describing excited state potential surfaces of conjugated organic molecules have been extensively investigated in Ref. 11.

Quantum Monte Carlo (QMC) is an alternative to conventional quantum chemical and density functional methods, and has been successfully employed to compute ground state properties of large molecules and solids. ${ }^{12}$ Compared to other theoretical approaches, QMC has the advantage that it can be applied to sufficiently large systems and still provide an ac- 
curate description of both dynamical and static electronic correlation. Despite the successful use of QMC for ground state problems, there is relatively little experience on its application to excited states. ${ }^{13-16}$ The recent QMC computation of excitation energies of large silicon nanostructures ${ }^{15}$ is very encouraging but the simple highest occupied molecular orbital-lowest unoccupied molecular orbital (HOMOLUMO) wave functions employed there are not likely to be adequate for photoactive systems due to the more complex nature of their electronic excitation.

To compare the accuracy of ROKS, TDDFT, and QMC in the study of photochemical processes, we compute the excitation to the lowest singlet state for a set of prototypical photoactive molecules: formaldimine $\left(\mathrm{CH}_{2} \mathrm{NH}\right)$, formaldehyde $\left(\mathrm{CH}_{2} \mathrm{O}\right)$, and a minimal protonated Schiff base model $\left(\mathrm{C}_{5} \mathrm{H}_{6} \mathrm{NH}_{2}^{+}\right)$of the retinal chromophore. For formaldimine and the protonated Schiff base model, we find that ROKS differs quantitatively and qualitatively from the other methods under consideration at low-symmetry molecular structures. While TDDFT excitation energies are fairly accurate in most situations, this method gives a qualitatively different result along a complete-active-space self-consistent-field (CASSCF) minimum energy path for the isomerization of the protonated Schiff base model. Finally, we find that QMC provides a reliable estimate of the lowest singlet excitation energies of the studied molecules, provided one makes an adequate and careful choice of the trial wave function. Although simple mean-field HOMO-LUMO Jastrow-Slater wave functions are not always adequate for these systems, we can recover accurate excitations energies by using a relatively small expansion in Slater determinants, whose orbitals and/or coefficients are reoptimized within QMC.

In Sec. II, we review the theoretical approaches employed in this work. The computational details are given in Sec. III and the numerical results are shown in Secs. IV A and IV B. Finally, in Sec. IV C, we discuss the sensitivity of the QMC results to the choice of the trial wave function.

\section{THEORETICAL METHODS}

We briefly review the theoretical methods used in this work for the computation of excited states, and refer for more details to the literature.

The ROKS method ${ }^{5,6}$ is a recent modification of the $\triangle \mathrm{SCF}$ approach used for the computation of multiplet splittings. ${ }^{17-20}$ In the ROKS approach, the energies of the states given by single determinants are not computed in separate calculations as in $\triangle \mathrm{SCF}$, but the linear combination corresponding to the desired state of pure symmetry is directly minimized under the constraint of orthogonality among the Kohn-Sham orbitals. In particular, the energy of an open shell singlet is estimated as $E(s)=2 E(m)-E(t)$, where $E(m)$ is the energy of the mixed singlet configuration, i.e., a single determinant having the open shell orbitals occupied with electrons of opposite spin, and $E(t)$ the energy of the corresponding triplet configuration. Within ROKS, the energy $E(s)$ is optimized using conventional ground state density functionals and a common set of orthogonal orbitals is used for both contributions. ${ }^{21}$
Both the $\triangle \mathrm{SCF}$ and ROKS approaches offer a practical recipe to the computation of excited states but they cannot be fully justified from a theoretical point of view and their validity must be empirically corroborated. An appealing feature of ROKS is that the method can be easily combined with $a b$ initio molecular dynamics and used to optimize the geometries in the excited state, access adiabatic excitations, and study the dynamics in the excited state. ${ }^{5,22-24}$ In general, even though the ROKS method tends to underestimate the excitation energies in particular for $\pi \rightarrow \pi^{*}$ transitions, ${ }^{22,25,26}$ it was shown to give a good description of the optimal geometries of the lowest excited states of small organic molecules, especially for $n \rightarrow \pi^{*}$ transitions. ${ }^{5,22}$

TDDFT is a different framework for the calculations of excited state properties which has become widely used in recent years. ${ }^{8}$ The method can handle large systems and, differently from $\triangle \mathrm{SCF}$ or ROKS, is formally exact even though, in practice, one has to resort to approximate exchange-correlation functionals. TDDFT has been extensively applied to the computation of vertical excitation energies since the calculation of forces within TDDFT is not straightforward and only recently a few implementation and applications of TDDFT to compute excited state geometries and adiabatic excitations have been published. ${ }^{11,27-29}$

Several quantum chemical approaches have been developed for a proper description of excited states. Methods such as multireference configuration interaction (MRCI) and CASPT2 rely on expanding, explicitly or implicitly, the wave function in Slater determinants. As the system size increases and the energies of the single-particle orbitals become closely spaced, the space of orbitals which must be included in the expansion to recover a significant fraction of electronic correlation grows enormously. Therefore, these techniques are very accurate but can only be applied to small systems. Even though CASPT2 was originally proposed as a method to compute excited state energies with an accuracy not better than $0.5 \mathrm{eV}$, it is now regarded as an approach which on average yields excitations in agreement with experiments to better than $0.2 \mathrm{eV} .{ }^{1}$ The method is quite sensitive to the construction of the active space which must include all important orbital excitations and is limited on current computers to a maximum of about 15 active orbitals.

Quantum Monte Carlo techniques ${ }^{12}$ is an alternative to density functional and conventional quantum chemistry approaches. While many studies have demonstrated the use and reliability of QMC for the description of ground state properties of molecular and solid systems, relatively little experience exists concerning its application to low-lying excited states. Recent studies of the excited states of methane, ethene, and small hydrogenated Si clusters indicate that the method is capable of reproducing the excitation energies of accurate quantum chemistry calculations. ${ }^{13,14,30}$ The QMC approach was also recently applied to the study of the excitations of large silicon nanoclusters, in combination with simple trial wave functions. ${ }^{15}$ QMC methods provide a stochastic solution of the Schrödinger equation: in diffusion Monte Carlo (DMC), the imaginary-time evolution operator $\exp (-\mathcal{H} \tau)$ is used to project out the ground state from a given trial wave function. ${ }^{31}$ To prevent the collapse to the 
bosonic ground state in fermionic systems, one works in the fixed-node approximation, that is, finds the best solution which has the same nodes as a given trial wave function. The solution is variational for the lowest state of a given spin symmetry belonging to a one-dimensional irreducible representation of the point group of the molecule. It is exact for any state if the nodes are exact. Therefore, if the nodal surface of the trial wave function is a good approximation to the excited state one, the fixed-node constraint can be used to access accurate excitation energies also of states which are not the lowest in their symmetry.

The trial many-body wave function employed in this paper is of the Slater-Jastrow form:

$$
\Psi_{\mathrm{T}}=\sum_{n} d_{n} D_{n}^{\uparrow} D_{n}^{\downarrow} \prod_{\alpha i j} J\left(r_{i j}, r_{i \alpha}, r_{j \alpha}\right),
$$

where $D_{n}^{\uparrow}$ and $D_{n}^{\downarrow}$ are Slater determinants of single particle orbitals for the up- and down-spin electrons, respectively, and the orbitals are represented using atomic Gaussian basis. The Jastrow factor correlates pairs of electrons $i$ and $j$ with each other, and with every nucleus $\alpha$, and different Jastrow factors are used to describe the correlation with different types of atoms. The parameters in the Jastrow factor are optimized within QMC using the variance minimization method. ${ }^{32}$ The Jastrow factor is positive and does not alter the nodal surface of the wave function which is instead fixed by the determinantal part. ${ }^{33}$ Particular attention must therefore be paid to the choice of the Slater component which is usually a linear combination of a small number of determinants. In the context of excited states, the CASSCF variant of the multiconfiguration self-consistent-field method (MCSCF) is particularly useful. These wave functions include all possible excitations for a given set of electrons within a chosen set of orbitals. When the excited state is not orthogonal to the ground state by symmetry, the determinantal component of the trial wave function is obtained in a state-average MCSCF approach, ${ }^{34}$ that is, by optimizing an average of the ground and excited state energies. Thus, the orbitals represent a compromise for describing both states.

Since the optimal orbitals and expansion coefficients in the presence of the Jastrow factor may differ from their optimal values in its absence, it is important to reoptimize them in the presence of the Jastrow component. To this end, we extended the energy fluctuation potential (EFP) method ${ }^{35}$ to simultaneously minimize the energy with respect to the orbitals and the expansion coefficients of a Slater-Jastrow wave function, as well as to handle state averaging necessary for excited states. ${ }^{30}$ In the absence of the Jastrow component, the method is analogous to the MCSCF technique for the lowest state of a given symmetry, and to a state-average MCSCF approach if the excited state of interest is not the lowest in its symmetry. Once the Jastrow factor is included, the orthogonality between the ground and excited states is only approximately preserved in the state-average EFP approach. The approach was tested for several singlet states of ethene and was shown to systematically improve the starting trial wave functions, correcting the initial excitation energies by as much as $0.5-0.6 \mathrm{eV}$ and yielding results in excellent agreement with experiments. $^{30}$

\section{COMPUTATIONAL DETAILS}

The ground-state DFT, and the excited-state ROKS and TDDFT calculations are performed with the Car-Parrinello molecular dynamics CPMD code. ${ }^{36,37}$ We employ the BLYP generalized gradient approximation for the exchange and correlation functional, ${ }^{38,39}$ the Goedecker pseudopotentials, ${ }^{40}$ an energy cutoff of 70 Ry for the plane-wave expansion, and a box size about $5 \AA$ larger then the size of the molecule. In order to avoid the inherent periodicity of a plane-wave calculation, we use the method described in Ref. 41, which solves the Poisson equation for nonperiodic boundary conditions, thus enabling the study of isolated molecules.

For formaldimine, the multireference configuration interaction singles and doubles (MR-CISD) calculations and the optimization of the excited state geometry within the stateaverage CASSCF method are performed with the COLUMBUS quantum chemistry program. ${ }^{42}$ Equal weights are used in the state-average CASSCF calculations for the optimization of the geometries. The reference space for MRCI is of six active electrons in six orbitals and the final MRCI energetics include Davidson corrections. It must be stressed that these MRCI calculations were performed with a moderate basis $((10 s 6 p 3 d) /[4 s 3 p 1 d]$ for carbon and nitrogen, and $(7 s 3 p) /[2 s 1 p]$ for hydrogen) and could certainly be improved. However, for the purpose of establishing the reliability of the other theoretical approaches, we consider the accuracy of the MRCI energetics to be sufficient.

For the QMC calculations, we use the CHAMP quantum Monte Carlo code ${ }^{43}$ and norm-conserving $s p$-nonlocal pseudopotentials for carbon, nitrogen and oxigen, generated in an all-electron Hartree-Fock calculation for the atoms. ${ }^{44}$ The orbitals in the determinantal component of the wave functions are expanded in the Gaussian basis sets $(11 s 11 p 2 d) /[4 s 4 p 2 d]$ for carbon, nitrogen, and oxigen, and $(10 s 2 p) /[3 s 2 p]$ for hydrogen. The basis sets are optimized at the Hartree-Fock (HF) level for formaldimine and formaldehyde. The determinantal part of the wave function, before reoptimization in QMC, is generated within HartreeFock, CASSCF or state-average CASSCF, using the quantum chemistry package GAMESS(US). ${ }^{45}$ Equal weights are used in the state-average CASSCF calculations, and in the state-average EFP optimization of the wave function. The Jastrow factor contains electron-electron, electron-nucleus and electron-electron-nucleus terms and is described in Ref. 46. For reasons of efficiency, most calculations are performed omitting the electron-electron-nucleus terms since the excitation energies for these systems computed with or without the three-body terms are the same within better than $0.1 \mathrm{eV}^{33}$ The diffusion Monte Carlo time step used for these molecules is $0.075 \mathrm{H}^{-1}$. Most of the QMC results presented below are obtained in diffusion Monte Carlo. Variational Monte Carlo (VMC) is also used to compute various expectation values of the trial Jastrow-Slater wave function.

\section{RESULTS}

The photosensitive molecules we investigate are schematically shown in Fig. 1. In formaldimine and formaldehyde, the lowest singlet excitation has predominantly a 
<smiles>CC(=O)C(C)(C)C</smiles>

Formaldimine

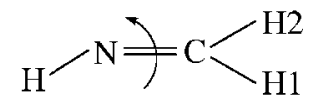

Protonated Schiff base

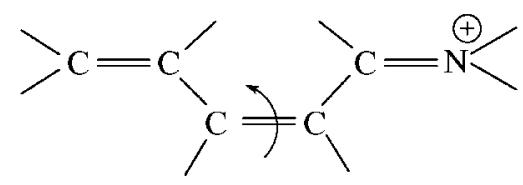

FIG. 1. Structure of the investigated molecules. In formaldimine and the protonated Schiff base model, the isomerization is around the bond indicated with an arrow. $\mathrm{H} 1 \mathrm{CNH}$ is the dyhedral angle varied in formaldimine.

$n \rightarrow \pi^{*}$ character and, in the protonated Schiff base model, a $\pi \rightarrow \pi^{*}$ character. The performance of the DFT-based approaches may differ for the two types of excitation, as has previously been stated for the ROKS method.

While QMC does not seem to be sensitive to the character of the excitation, a different complication is encountered when performing excited state QMC calculations. If the excited state of interest is the lowest state of a given spin symmetry belonging to a one-dimensional irreducible representation, the DMC energy is variational. In all other cases, $\mathrm{DMC}$ is no longer variational and the quality of the trial wave function becomes increasingly important. The vertical and adiabatic excitations of formaldimine and formaldehyde belong to the first category while the excitations of the minimal protonated Schiff base model and of formaldimine along its isomerization path belong to the other case.

\section{A. Formaldimine and formaldehyde}

In the $n \rightarrow \pi^{*}$ excitation of formaldimine and formaldehyde, a lone-pair electron is transferred to a $\pi^{*}$ antibonding orbital. The excitation is almost purely of the HOMOLUMO type and has therefore been considered ideal for the ROKS approach, ${ }^{5}$ which was also used to study the excited state cis-trans isomerization of formaldimine in a BornOppenheimer molecular dynamics simulation ${ }^{5}$ and, more recently, in a nonadiabatic Car-Parrinello dynamics. ${ }^{23}$

In Table I, we list the vertical and adiabatic lowest singlet excitation energies, evaluated using ROKS, TDDFT, and DMC. The vertical excitations are computed on the ground state DFT geometries, while the adiabatic excitations on the geometries optimized in the excited state using ROKS. The adiabatic geometry of formaldehyde is known experimentally and is well reproduced by ROKS. ${ }^{5}$ Vertical and adiabatic transitions are underestimated by ROKS by as much as $0.5 \mathrm{eV}$, while the TDDFT results are in reasonable agreement with experiments. These findings are consistent with previous ROKS calculations for both molecules, ${ }^{5}$ and with TDDFT calculations of the vertical ${ }^{51}$ and adiabatic ${ }^{28}$ excitations of formaldehyde.

The DMC excitations are obtained using a comparable description of the ground and excited states. A onedeterminant trial wave function is used for the ground state, and a two-determinant singlet wave function for the excited state, corresponding to a single excitation from the doubly occupied $n$ HOMO to the $\pi^{*}$ LUMO. The starting orbitals in the determinantal component of the QMC wave function are from a HF calculation in the ground state, and a twodeterminant MCSCF calculation in the excited state. For both states, all orbitals are subsequently optimized in the presence of the Jastrow factor with the EFP method. For formaldehyde, the DMC excitation energies are slightly higher than available experimental numbers and results from highly correlated quantum chemistry calculations, which however show a significant spread. The vertical excitation energies computed with quantum chemistry techniques ${ }^{52-55}$ range between $3.98 \mathrm{eV}$ from equation of motion-coupled cluster (EOM-CC) and $4.19 \mathrm{eV}$ from MRCI, ${ }^{55}$ while MRCI calculations for the adiabatic transition ${ }^{56}$ yield an excitation energy of 3.60-3.66 eV. For formaldimine, the DMC vertical and adiabatic excitations are in good agreement with MRCI calculations. ${ }^{57}$

While the success of DMC in describing these vertical and adiabatic excitations is encouraging, it is important to assess its performance when variationality is lost as happens along the low-symmetry isomerization path induced by the excitation. We therefore consider the prototypical case of the isomerization of formaldimine around the $\mathrm{C}-\mathrm{N}$ double bond. The isomerization path is constructed by constraining the torsional angle $\mathrm{H} 1 \mathrm{CNH}$ (see Fig. 1) at values between $0^{\circ}$ and $90^{\circ}$, with increments of $15^{\circ}$. The molecule has $C_{s}$ symmetry at $0^{\circ}$ and $90^{\circ}$, and no symmetry at intermediate angles.

In Fig. 2, we show the ROKS, TDDFT, DMC, and MRCI excitation energies on the excited state geometries optimized with ROKS at constrained torsional angles. The

TABLE I. Vertical and adiabatic lowest singlet excitation energies in eV for formaldehyde and formaldimine, calculated within ROKS, TDDFT, and DMC. The numbers in parentheses are the statistical errors on the DMC results.

\begin{tabular}{llcccc}
\hline \hline System & Excitation & ROKS & TDDFT & DMC & Expt. \\
\hline $\mathrm{CH}_{2} \mathrm{O}$ & Vertical & 3.58 & 3.90 & $4.24(2)$ & $3.94^{\mathrm{a}}, 4.07^{\mathrm{b}}, 4.2^{\mathrm{c}}$ \\
& Adiabatic & 3.13 & 3.51 & $3.74(2)$ & $3.50^{\mathrm{d}}$ \\
$\mathrm{CH}_{2} \mathrm{NH}$ & Vertical & 4.63 & 5.34 & $5.32(2)$ & $5.0-5.4^{\mathrm{b}}$ \\
& Adiabatic & 2.85 & 3.23 & $3.21(2)$ & $\cdots$ \\
\hline \hline
\end{tabular}

${ }^{\mathrm{a}}$ Reference 47.

${ }^{\mathrm{b}}$ Reference 48 .

${ }^{\mathrm{c}}$ Reference 49.

${ }^{\mathrm{d}}$ Reference 50. 


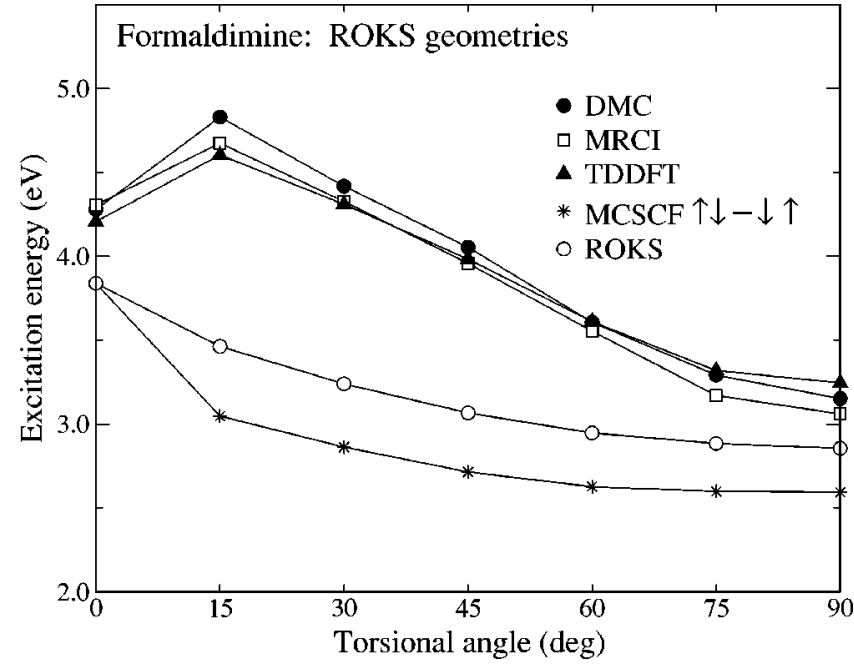

FIG. 2. Lowest-singlet excitation energies of formaldimine in $\mathrm{eV}$ calculated with ROKS, TDDFT, MRCI, and DMC on the excited state geometries optimized with ROKS at constrained torsional angles. The excitations computed within a two-determinant MCSCF calculation $(\uparrow \downarrow-\downarrow \uparrow)$ are also shown. The statistical error on the DMC results is smaller than the size of the symbols.

excitation energies are given with respect to the ground state energy consistently computed within the same approach on the DFT ground state geometry at zero torsional angle. The DMC excited state energies are obtained with a trial wave function from a state-average CASSCF with an active space of six electrons in six orbitals, whose expansion coefficients are then reoptimized in the presence of the Jastrow factor with a state-average EFP method. The DMC ground state energy at zero torsional angle is computed with an unoptimized HF determinantal component. The DMC excitations are in very good agreement with the MRCI values, with a maximum deviation of $0.13 \mathrm{eV}$ along the curve.

While the TDDFT excitations agree with the MRCI values to better than $0.2 \mathrm{eV}$, the ROKS curve differs significantly. In particular, MRCI gives a barrier to isomerization along the geometries corresponding to an energy minimum path in ROKS. One can possibly understand the behavior of ROKS by looking at the results obtained with a twodeterminant MCSCF (without state-average) approach along the same path. As shown in Fig. 2, the two-determinant MCSCF curve is qualitatively very similar to the ROKS curve. For the two-determinant MCSCF calculation, only the orthogonality constraint on the open shell orbitals keeps the wave function from completely collapsing to the ground state. By analogy, the ROKS approach is likely to suffer from the same problem whenever ground and excited states do not belong to different irreducible representations. ${ }^{22}$

To further investigate the constraint isomerization path of formaldimine, we optimize the geometries using the excited-state forces from a state-average CASSCF approach with an active space of six electrons in six orbitals. As already pointed out in early MRCI studies by BonačićKoutecký et al. ${ }^{58}$ to properly describe the isomerization of formaldimine, one should map the potential energy surface with respect to the $\mathrm{CNH}$ valence angle and a properly symmetrized dyhedral angle. However, the path obtained within

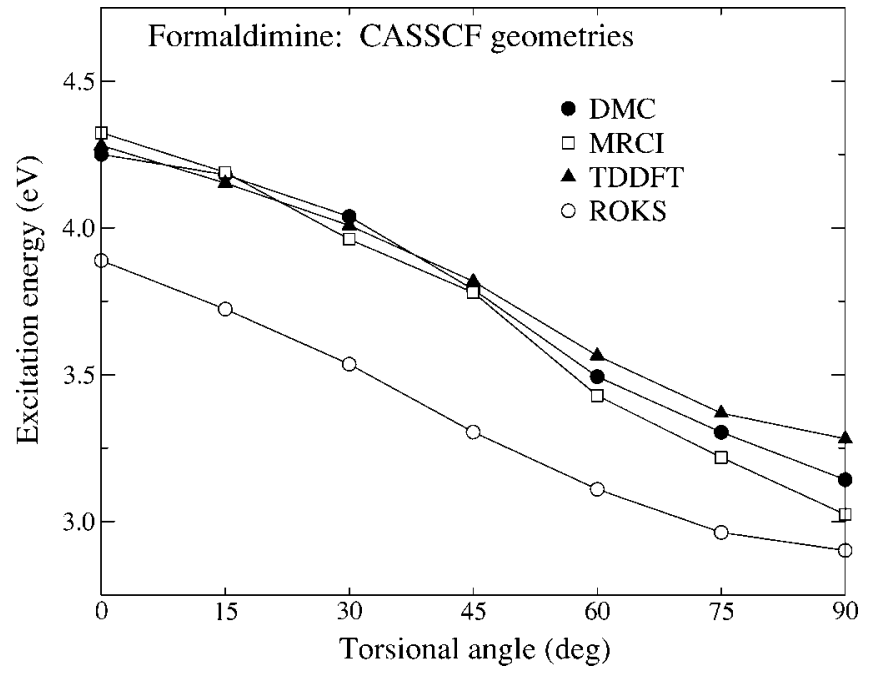

FIG. 3. Lowest-singlet excitation energies of formaldimine in eV calculated with ROKS, TDDFT, MRCI, and DMC on the excited state geometries optimized using a state-average CASSCF (see text) at constrained torsional angles.

CASSCF by only constraining the $\mathrm{H} 1 \mathrm{CNH}$ dyhedral angle is reasonably close to the optimal path. We find that the main difference between the ROKS and CASSCF paths is in the behavior of the angle $\mathrm{CNH}$ which, in ROKS, takes his final value corresponding to a torsional angle of 90 degrees as soon as the molecule is displaced from planarity.

The excitations computed with TDDFT, ROKS, DMC, and MRCI on the CASSCF geometries are shown in Fig. 3. The DMC calculations are performed with the same type of wave function previously used for the ROKS path. The energy barrier to isomerization present in Fig. 2 disappears in MRCI as this barrier was an artifact of using the geometries optimized within ROKS. The DMC excitation energies are very close to the MRCI values with a maximum difference of $0.1 \mathrm{eV}$ along the CASSCF path. TDDFT is in reasonable agreement with QMC also along this path. For the CASSCF geometries, ROKS calculations produce a curve of similar shape as those obtained with the other methods, but significantly shifted toward lower energies.

\section{B. Protonated Shiff base model}

The $\mathrm{C}_{5} \mathrm{H}_{6} \mathrm{NH}_{2}^{+}$protonated Shiff base molecule represents a minimal model for studying the retinal photoisomerization process in rhodopsin. Given its relevance and combined simplicity, this molecule is ideal for accessing the relative accuracy of different theoretical approaches. Moreover, this model has been extensively studied within CASPT2 using geometries optimized in the excited state with CASSCF (Refs. 2 and 3) and, more recently, with CASPT $2{ }^{4}$

Since ROKS was previously employed to study the excited state of the full retinal chromophore including relevant parts of the protein environment, ${ }^{7}$ it is interesting to use the same approach to optimize the structure of this simpler model. In Fig. 4, we show the ROKS, TDDFT, and DMC energetics computed on the geometries optimized within ROKS along the relevant isomerization coordinate repre- 


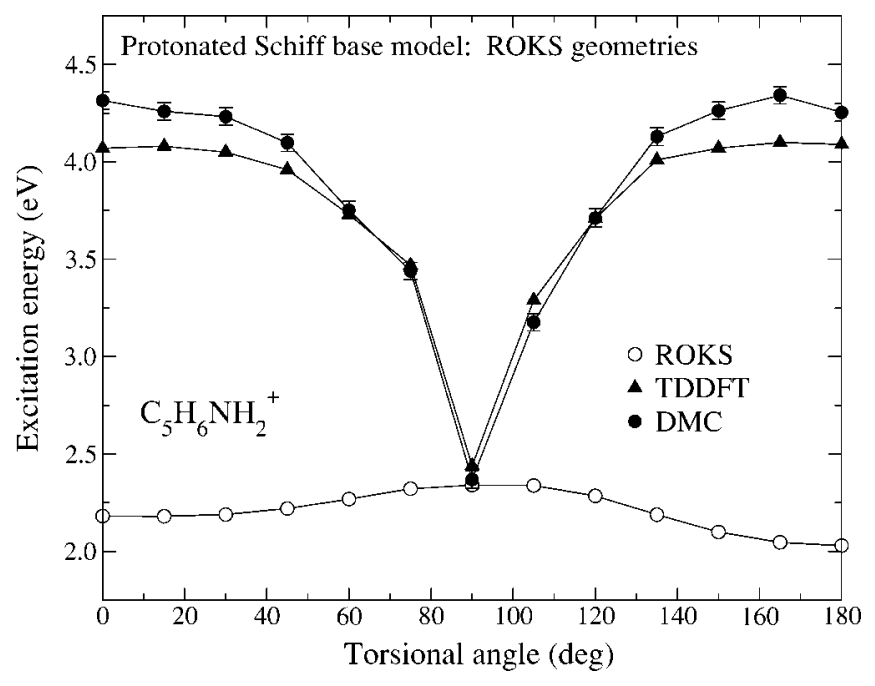

FIG. 4. Lowest-singlet excitations energies for the protonated Schiff base model in eV calculated with ROKS, TDDFT, and DMC on the excited state geometries optimized with ROKS at constrained torsional angles. The excitation energies are given with respect to the ground state energy consistently computed within the same approach on the DFT ground state cis-geometry at zero torsional angle.

sented by the torsional angle around the central C-C double bond (see Fig. 1). When optimizing the excited state geometry with ROKS, the molecule remains planar and the main effect of the excitation is a considerable lengthening of the double bonds and a shortening of the single bonds, thus reversing the conjugation of the molecule. The ROKS potential energy surface along the torsion is quite flat with a maximum at $90^{\circ}$. This behavior is qualitatively different from the CASSCF and CASPT2 energy profile, ${ }^{2}$ where the torsion accelerates the system towards the conical intersection, thus spontaneously inducing the photoisomerization. Therefore, while the ROKS method shows a stretching mode starting from the Franck-Condon region similar to the CASSCF result, it does not reproduce the qualitative shape of the excited state CASSCF potential energy surface along the torsional mode.

The DMC excited state energies in Fig. 4 are computed on the ROKS geometries with a trial wave function from a state-average CASSCF with an active space of six electrons in six orbitals, whose expansion coefficients are then reoptimized in the presence of the Jastrow factor with a stateaverage EFP method. The TDDFT excitation energies are higher than the ROKS values by as much as $2 \mathrm{eV}$, and in agreement with the DMC results to better than $0.2 \mathrm{eV}$. The TDDFT and DMC potential energy curves have a very different shape than the one obtained within ROKS. In the protonated Schiff base model, the ground and excited states belong to the same irreducible representation both when the molecule is planar and twisted. The behavior of ROKS can possibly be explained as due to a contamination of the excited state with the ground state as in the case of twisted formaldimine.

To allow for a comparison with existing CASPT2 calculations on this model, we consider three geometries which were optimized in Ref. 2 within state-average CASSCF and where the CASPT2 energies are also available. These struc-
TABLE II. Lowest-singlet excitation energies for the protonated Schiff base model in $\mathrm{eV}$, calculated with TDDFT, CASPT2, and DMC on the ground state cis-configuration (FC), on the geometry (HM) which demarcates where torsion becomes dominant, and on the conical intersection (CI). The CASSCF geometries and the CASPT2 numbers are from Ref. 2. The excitation energies are given with respect to the ground state energy consistently computed within the same approach on the CASSCF ground state cisgeometry at zero torsional angle.

\begin{tabular}{lccc}
\hline \hline Geometry & TDDFT & CASPT2 & DMC \\
\hline FC & 3.90 & 4.02 & $4.38(5)$ \\
HM & 4.12 & 3.71 & $4.22(5)$ \\
CI & 2.18 & 2.19 & $2.58(5)$ \\
\hline \hline
\end{tabular}

tures correspond to the ground state cis-configuration where the Franck-Condon (FC) excitation is computed, to the geometry which demarcates where torsion becomes dominant along the isomerization path (denoted with HM in Ref. 2), and to the $S_{0} / S_{1}$ conical intersection (CI). Without a direct comparison with experimental data, it is difficult to access the accuracy of these excited state structures: for instance, when compared to geometries optmized with CASPT2, the CASSCF structures are very similar at the conical intersection but significantly different at constrained planar symmetry. 4

In Table II, we list the TDDFT, CASPT2, and DMC excitation energies at the FC, HM, and CI geometries. The DMC calculations are performed with the same type of wave function previously used for the ROKS path. The use of larger active spaces (six electrons in nine orbitals or eight electrons in eight orbitals) and the reoptimization of the active orbitals with the state-average EFP method yield DMC energies compatible to better than $0.1 \mathrm{eV}$. While the CASPT2 and QMC results are qualitatively similar, the CASPT2 energies are lower than the QMC values by as much as $0.5 \mathrm{eV}$. The order of the TDDFT excitation energies at the FC and HM configurations are instead reversed with respect to the DMC values: the TDDFT excitation is lower at FC than at HM, so TDDFT gives a barrier to isomerization along the CASSCF path. A valid question is whether this barrier survives when using an excited state path fully optimized within TDDFT. Recently, it has been shown that the TDDFT gradient for various protonated Schiff base models differs qualitatively from that of CASSCF/CASPT2, driving the system from the FC point to a planar fictitious stationary point. ${ }^{11}$

Finally, in order to further compare TDDFT and QMC, we generate a set of geometries for $\mathrm{C}_{5} \mathrm{H}_{6} \mathrm{NH}_{2}^{+}$by starting from the HM structure of Ref. 2 and increasing the torsional angle up to about $90^{\circ}$ while keeping all the other internal coordinates fixed. In Fig. 5, we show the TDDFT and DMC energies, and the CASPT2 results at FC and HM. Along the torsional path after HM, TDDFT, and DMC follow closely each with a larger deviation at the end of the path.

\section{Sensitivity of DMC to the trial wave function}

Using as examples the vertical excited state and the adiabatic isomerization path of formaldimine, we demonstrate 


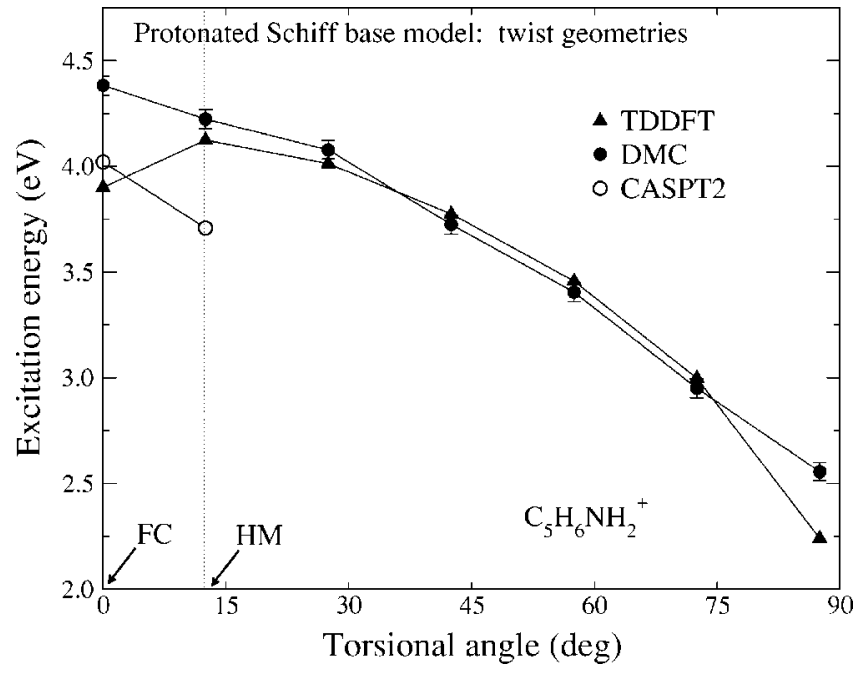

FIG. 5. Excitation energies for the protonated Schiff base model in eV, calculated with TDDFT and DMC on a set of geometries generated by rigidly increasing the torsional angle, from the HM configuration. The TDDFT, DMC, and CASPT2 (Ref. 2) energies at FC and HM are also given.

how sensitive the QMC energies are to the choice of the wave function and how this sensitivity can vary along the excited state potential energy curve.

The vertical lowest-singlet excited state of formaldimine does not have a strong multiconfigurational character, and a two-determinant Jastrow-Slater wave function to preserve spin symmetry is found to be sufficient for this particular state. The QMC energies are variational since this excited state is the lowest in its symmetry, and orthogonality between ground and excited state is automatically ensured. For the ground state, a single determinant wave function gives an adequate description. In Table III, we show the VMC and fixed-node DMC energies determined with different choices of orbitals in the determinantal component of the wave function. The starting trial wave function uses orbitals obtained from a $\mathrm{HF}$ and a two-determinant MCSCF calculations for the ground and excited state, respectively. By optimizing the orbitals with the EFP method, the VMC energy drops by 10 mhartree in the ground state and by 15 mhartree in the excited state. However, the gain in the DMC energies is only of a few millihartree and is actually more significant in the ground state. The resulting DMC excitation energy is only slightly higher as a result of the optimization.

TABLE III. VMC and DMC ground state $\left(S_{0}\right)$ and lowest-singlet excited state $\left(S_{1}\right)$ energies in Hartree for formaldimine, calculated at the ground state geometry. In the Jastrow-Slater wave function, a single determinant is used for the ground state and two determinants for the excited state. The DMC excitation energies in $\mathrm{eV}$ are computed using unoptimized (HF for $S_{0}$ and MCSCF for $S_{1}$ ) and optimized (EFP) orbitals for both states.

\begin{tabular}{lcccc}
\hline \hline State & Orbitals & $E_{\mathrm{VMC}}$ & $E_{\mathrm{DMC}}$ & $\Delta E(\mathrm{eV})$ \\
\hline$S_{0}$ & $\mathrm{HF}$ & $-17.2973(4)$ & $-17.3685(5)$ & $\ldots$ \\
& $\mathrm{EFP}$ & $-17.3082(4)$ & $-17.3726(5)$ & $\ldots$ \\
$S_{1}$ & $\mathrm{MCSCF}$ & $-17.1185(4)$ & $-17.1756(5)$ & $5.25(2)$ \\
& $\mathrm{EFP}$ & $-17.1334(4)$ & $-17.1772(4)$ & $5.32(2)$ \\
\hline \hline
\end{tabular}

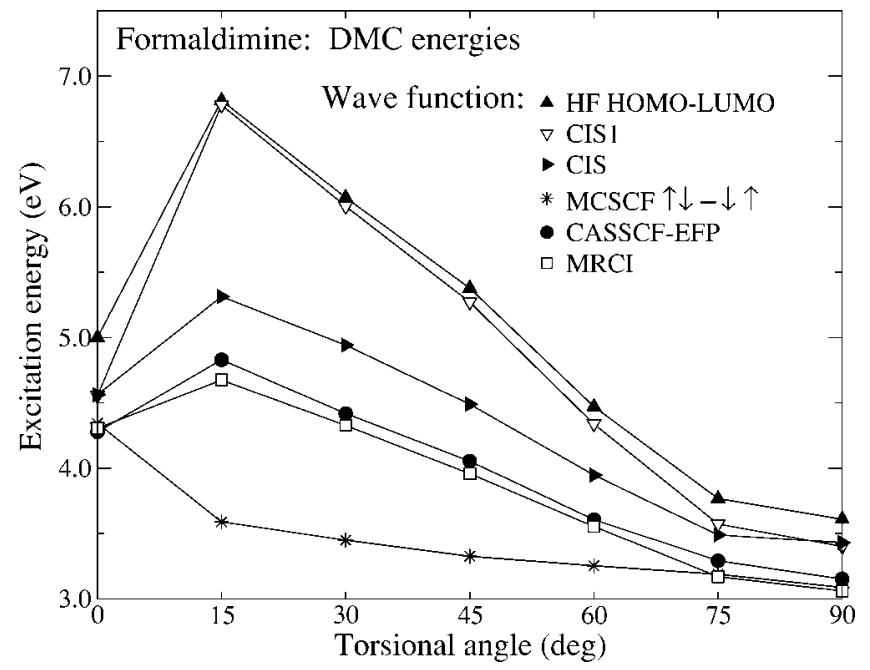

FIG. 6. DMC lowest-singlet excited state energies of formaldimine in $\mathrm{eV}$, computed on the ROKS geometries at various torsional angles, using different trial wave functions. See text for more details.

Along the isomerization path of formaldimine, orthogonality between ground and excited state is no longer maintained and a higher sensitivity of the QMC results to the trial wave function may be expected than in the case of the vertical excitation. In Fig. 6, we compare the DMC excitation energies along the ROKS isomerization path of formaldimine for different choices of wave functions previously employed in other QMC studies of excited states. At $0^{\circ}$ and $90^{\circ}$ torsional angles where the energy is variational due to symmetry, the spread of the DMC energies due to the use of different wave functions is significantly smaller than at intermediate angles. A simple two-determinant HOMO-LUMO wave function with HF orbitals shows a discrepancy as large as $1.5 \mathrm{eV}$ with our best DMC results obtained with a six electrons in six orbitals CASSCF wave function whose CI coefficients have been reoptimized with the state-average EFP method. The wave function denoted with "CIS1" includes all single excitations from the HOMO, and can be resummed to two determinants, where only the LUMO has therefore been changed with respect to the HF orbitals. The CIS1 energies represent an improvement at the end points of the path but remain as poor as those obtained with a HOMOLUMO wave function at almost all other angles. If all single excitations are included in a configuration integration singles (CIS) wave function, the excitation energies are significantly closer to the CASSCF-EFP results along the whole path, with an almost constant discrepancy of $0.3-0.5 \mathrm{eV}$. Finally, one could be tempted to use a two-determinant wave function obtained in a MCSCF calculation (without stateaverage). While this wave function performs well at $0^{\circ}$ and $90^{\circ}$ where ground and excited states are orthogonal by symmetry, it represents a poor starting point at low-symmetry configurations as already discussed in Sec. IV A, yielding DMC energies which are obviously non variational.

Finally, the effect of truncating the determinantal expansion according to a threshold on the coefficients is investigated. It is indeed customary in QMC to apply a threshold for computational efficiency, justified by the very different 
TABLE IV. VMC and DMC lowest-singlet excited state energies for formaldimine, computed on the ROKS geometries at various torsional angles. Different determinantal components are used in the trial wave functions, with thresholds of 0.1 and 0.01 on the expansion in symmetry-adapted configuration state functions from a state-average CASSCF, and with CASSCF and EFP-optimized expansion coefficients.

\begin{tabular}{|c|c|c|c|}
\hline $\begin{array}{l}\text { Threshold } \\
\text { Coefficients }\end{array}$ & $\begin{array}{c}0.1 \\
\text { CASSCF }\end{array}$ & $\begin{array}{c}0.01 \\
\text { CASSCF }\end{array}$ & $\begin{array}{l}0.01 \\
\text { EFP }\end{array}$ \\
\hline Angle (deg) & \multicolumn{3}{|c|}{ Number of determinants } \\
\hline 0 & 4 & 42 & 23 \\
\hline 30 & 9 & 132 & 46 \\
\hline 60 & 8 & 108 & 54 \\
\hline 90 & 4 & 71 & 35 \\
\hline Angle (deg) & \multicolumn{3}{|c|}{ VMC energies (Hartree) } \\
\hline 0 & $-17.158(1)$ & $-17.152(1)$ & $-17.165(1)$ \\
\hline 30 & $-17.149(1)$ & $-17.144(1)$ & $-17.158(1)$ \\
\hline 60 & $-17.180(1)$ & $-17.178(1)$ & $-17.190(1)$ \\
\hline 90 & $-17.200(1)$ & $-17.193(1)$ & $-17.205(1)$ \\
\hline Angle (deg) & \multicolumn{3}{|c|}{ DMC energies (Hartree) } \\
\hline 0 & $-17.2099(5)$ & $-17.2063(5)$ & $-17.2113(4)$ \\
\hline 30 & $-17.2027(5)$ & $-17.2000(5)$ & $-17.2062(4)$ \\
\hline 60 & $-17.2338(5)$ & $-17.2313(5)$ & $-17.2360(4)$ \\
\hline 90 & $-17.2502(4)$ & $-17.2474(5)$ & $-17.2527(4)$ \\
\hline
\end{tabular}

role of the reference wave function in QMC compared to conventional quantum chemistry methods. A smaller number of determinants is needed in a Jastrow-Slater wave function since the reference wave function does not define the singleparticle excitation space for the description of dynamical correlation as is the case for a method like MRCI. Moreover, one hopes that the effect of determinants with a small coefficient on the nodal surface of the total wave function is not significant.

In Table IV, we show the VMC and DMC excited state energies for formaldimine, computed on the ROKS geometries at various torsional angles when applying two different thresholds on the expansion coefficients in symmetryadapted configuration state functions. The starting trial wave function is obtained from a state-average CASSCF with an active space of six electrons in six orbitals. As the threshold is lowered from 0.1 to 0.01 , both VMC and DMC energies become higher at all angles. Since at $0^{\circ}$ and $90^{\circ}$ the energies are variational due to symmetry, one is unequivocally aiming at obtaining the lowest possible energy at those geometries and one would have expected a lowering of the energy by including more configurations. This indicates that the result is strongly dependent on the chosen threshold if one does not reoptimize the determinantal expansion in the presence of the Jastrow factor. The coefficients of the starting CASSCF wave function are therefore reoptimized with the stateaverage EFP method. The natural orbitals of the averaged single-particle density matrix of the reoptimized expansions are here used to obtain a more compact wave function, and a threshold of 0.01 is then applied. The corresponding VMC and DMC energies are also shown in Table IV. At all angles, the VMC energies for the reoptimized wave function are lower than the values obtained using the original CASSCF coefficients with respect to the same threshold. Moreover, the optimal energies are also systematically better than the VMC values obtained with a threshold of 0.1. In Table IV, we also list the number of determinants with coefficients greater than the chosen threshold. As expected, due to the inclusion of dynamical correlation through the Jastrow factor, the wave function becomes more compact as an effect of the reoptimization. The DMC energies behave similarly to the VMC values with respect to both threshold and reoptimization. The excitation energies obtained in DMC with the reoptimized wave function are in excellent agreement with the MRCI values as shown in Sec. IV A. If a threshold of 0.1 is used when reoptimizing the expansion coefficients in a state-average EFP method, there is no improvement in the QMC energies compared to the values obtained with the original CASSCF coefficients and the same threshold.

Finally, if the orbitals are optimized with the stateaverage EFP approach and a threshold of 0.1, both VMC and DMC energies improve and became equal to the values obtained with the CASSCF-EFP with 0.01 threshold. For instance, for a torsional angle of $30^{\circ}$, the optimization of the orbitals yields a VMC and a DMC energy of $-17.156(1)$ and -17.2071(4) hartree, respectively. We want to stress that there is in general no justification for using a threshold as high as 0.1 and the apparent agreement with the optimized energies is here a fortunate case.

\section{CONCLUSIONS}

Using TDDFT, ROKS, and QMC, we have investigated the lowest-singlet excitation energies along various isomerization paths for the following representative photoactive molecules: formaldehyde, formaldimine and a minimal protonated Schiff base model $\mathrm{C}_{5} \mathrm{H}_{6} \mathrm{NH}_{2}^{+}$.

We show that fixed-node diffusion Monte Carlo can give accurate excitation energies, provided a careful choice of QMC trial wave function is made. While simple HOMOLUMO trial wave functions are not always adequate to describe the excited states of these photoactive molecules, accurate results are recovered when using a relatively small expansion in Slater determinants, whose coefficients and/or orbitals are reoptimized in the presence of the Jastrow factor with the EFP method.

TDDFT yields excitation energies which are generally in reasonable agreement with the QMC results. However, the TDDFT energies for the minimal model of the retinal chromophore are in qualitative disagreement with QMC and CASPT2, giving a barrier to isomerization along the CASSCF minimal energy path.

We find that the ROKS method does not produce reliable results for the excited-state potential energy surface at lowsymmetry configurations. The major source of error in the ROKS approach seems to be the contamination of the excited state with the ground state. For example, ROKS predicts an energy barrier to isomerization with a maximum at $90^{\circ}$ along the relevant torsional angle of the minimal protonated Schiff base model of the retinal chromophore, while TDDFT and QMC show a minimum at this point. Therefore, even though the ROKS method is appealing for its simplicity 
in computing forces, it should be generally used with caution in excited-state molecular dynamics simulations.

Note added in proof. We thank N. L. Doltsinis for pointing out to us that the planar geometry of formaldimine at zero torsional angle used in Fig. 2 is only a local minimum in the ROKS potential energy surface. We have since verified that ROKS yields a pyramidalized structure at zero torsional angle with an excitation energy which is lower by about 0.08 eV. However, this geometrical change does not significantly affect the other results in this paper.

\section{ACKNOWLEDGMENTS}

We thank J. Hutter for helpful discussions and C. J. Umrigar for a critical reading of the manuscript. This work was in part funded by the Stichting voor Fundamenteel Onderzoek der Materie (FOM), which is financially supported by the Nederlandse Organisatie voor Wetenschappelijk Onderzoek (NWO).

${ }^{1}$ B. O. Roos, K. Andersson, M. P. Fülscher, P.-A. Malmqvist, and L. Serrano-Andrés, Advances in Chemical Physics, edited by I. Prigogine and S. A. Rice (Wiley, New York, 1996), pp. 219-331, Vol. XCIII.

${ }^{2}$ M. Garavelli, P. Celani, F. Bernardi, M. A. Robb, and M. Olivucci, J. Am. Chem. Soc. 119, 6891 (1997).

${ }^{3}$ R. Gonzalez-Luque, M. Garavelli, F. Bernardi, M. Merchan, M. A. Robb, and M. Olivucci, Proc. Natl. Acad. Sci. U.S.A. 97, 9379 (2000).

${ }^{4}$ C. S. Page and M. Olivucci, J. Comput. Chem. 24, 298 (2003).

${ }^{5}$ I. Frank, J. Hutter, D. Marx, and M. Parrinello, J. Chem. Phys. 108, 4060 (1998).

${ }^{6}$ M. Filatov and S. Shaik, Chem. Phys. Lett. 288, 689 (1998); J. Chem. Phys. 110, 116 (1999).

${ }^{7}$ C. Molteni, I. Frank, and M. Parrinello, J. Am. Chem. Soc. 121, 12177 (1999); Comput. Mater. Sci. 20, 311 (2001).

${ }^{8}$ M. E. Casida, in Recent Advances in Density Functional Methods, edited by D. P. Chong (World Scientific, Singapore, 1995), p. 155, Pt. I.

${ }^{9}$ Z.-L. Cai, K. Sendt, and J. R. Reimers, J. Chem. Phys. 117, 5543 (2002).

${ }^{10}$ A. Dreuw, J. L. Weisman, and M. Head-Gordon, J. Chem. Phys. 119, 2943 (2003).

${ }^{11}$ M. Wanko, M. Garavelli, F. Bernardi, T. A. Niehaus, T. Frauenheim, M. Elstner, J. Chem. Phys. 120, 1674 (2004).

${ }^{12}$ W. M. C. Foulkes, L. Mitas, R. J. Needs, and G. Rajagopal, Rev. Mod. Phys. 73, 33 (2001).

${ }^{13}$ J. C. Grossman, M. Rohlfing, L. Mitas, S. G. Louie, and M. L. Cohen, Phys. Rev. Lett. 86, 472 (2001).

${ }^{14}$ A. R. Porter, O. K. Al-Mushadani, M. D. Towler, and R. J. Needs, J. Chem. Phys. 114, 7795 (2001); A. R. Porter, M. D. Towler, and R. J. Needs, Phys. Rev. B 64, 035320 (2001).

${ }^{15}$ A. Puzder, A. J. Williamson, J. C. Grossman, and G. Galli, Phys. Rev. Lett. 88, 097401 (2002); A. J. Williamson, J. C. Grossman, R. Q. Hood, A. Puzder, and G. Galli, ibid. 89, 196803 (2002).

${ }^{16}$ A. Aspuru-Guzik, O. El Akramine, J. C. Grossman, and W. A. Lester, Jr., J. Chem. Phys. 120, 3049 (2004).

${ }^{17}$ U. von Barth, Phys. Rev. A 20, 1693 (1979); Phys. Scr. 21, 585 (1980).

${ }^{18}$ T. Ziegler, A. Rauk, and B. J. Baerends, Theor. Chim. Acta 43, 261 (1977).

${ }^{19}$ O. Gunnarson and R. O. Jones, J. Chem. Phys. 72, 5357 (1980).

${ }^{20}$ C. Daul, Int. J. Quantum Chem. 52, 867 (1994).

${ }^{21}$ Recently, it was suggested that the original implementation of the ROKS method might not always lead to the minimal energy and a revised algorithm was proposed. See Ref. 25.

${ }^{22}$ M. Odelius, D. Laikov, and J. Hutter, J. Mol. Struct.: THEOCHEM 630, 163 (2003)
${ }^{23}$ N. L. Doltsinis and D. Marx, Phys. Rev. Lett. 88, 166402 (2002).

${ }^{24}$ S. Grimm, C. Nonnenberg, and I. Frank, J. Chem. Phys. 119, 11585 (2003).

${ }^{25}$ S. Grimm, C. Nonnenberg, and I. Frank, J. Chem. Phys. 119, 11574 (2003).

${ }^{26}$ H. Langer and N. L. Doltsinis, J. Chem. Phys. 118, 5400 (2003).

${ }^{27}$ C. Van Caillie and R. D. Amos, Chem. Phys. Lett. 317, 159 (2000).

${ }^{28}$ F. Furche and R. Ahlrichs, J. Chem. Phys. 117, 7433 (2002).

${ }^{29}$ J. Hutter, J. Chem. Phys. 118, 3928 (2003).

${ }^{30}$ F. Schautz and C. Filippi, J. Chem. Phys. 120, 10931 (2004).

${ }^{31}$ P. J. Reynolds et al., J. Chem. Phys. 77, 5593 (1982); L. Mitas, E. L. Shirley, and D. M. Ceperley, ibid. 95, 3467 (1991); C. J. Umrigar, M. P. Nightingale, and K. J. Runge, ibid. 99, 2865 (1993).

${ }^{32}$ C. J. Umrigar, K. G. Wilson, and J. W. Wilkins, Phys. Rev. Lett. 60, 1719 (1988).

${ }^{33}$ If nonlocal pseudopotentials are used, the trial wave function including the Jastrow factor is used to localize the pseudopotential so that the DMC energy will depend on the Jastrow factor.

${ }^{34}$ H.-J. Werner and P. J. Knowles, J. Chem. Phys. 82, 5053 (1985); A. Spielfiedel, N. G. Feautrier, P. R. Chambaud, and H. J. Werner, Chem. Phys. Lett. 183, 16 (1991); K. Docken and J. Hinze, J. Chem. Phys. 57, 4928 (1972).

${ }^{35}$ C. Filippi and S. Fahy, J. Chem. Phys. 112, 3523 (2000); F. Schautz and S. Fahy, ibid. 116, 3533 (2002); D. Prendergast, D. Bevan, and S. Fahy, Phys. Rev. B 66, 155104 (2002).

${ }^{36}$ R. Car and M. Parrinello, Phys. Rev. Lett. 55, 2471 (1985).

${ }^{37}$ We used the CPMD code, version 3.6, developed by J. Hutter et al., Copyright IBM Corp 1990-2001, Copyright MPI für Festkörperforschung Stuttgart 1997-2001.

${ }^{38}$ A. D. Becke, J. Chem. Phys. 84, 4524 (1986).

${ }^{39}$ C. Lee, W. Yang, and R. Parr, Phys. Rev. B 37, 785 (1988).

${ }^{40}$ S. Goedecker, M. Teter, and J. Hutter, Phys. Rev. B 54, 1703 (1996).

${ }^{41}$ G. J. Martyna and M. E. Tuckerman, J. Chem. Phys. 110, 2810 (1999).

${ }^{42}$ We used the code Columbus, release 5.8 , developed by H. Lischka, R. Schepard, I. Shavitt et al.; H. Lischka, R. Schepard, F. B. Brown, and I. Shavitt, Int. J. Quantum Chem. S15, 91 (1981).

${ }^{43}$ C. J. Umrigar and C. Filippi, Cornell Holland Ab-initio Materials Package (СHAMP). The code can be used for finite and extended systems. See http:// www.lorentz.leidenuniv.nl/filippi/champ.html

${ }^{44}$ We used the code of E. Shirley to generate norm-conserving Hartree-Fock pseudopotential with the construction by D. Vanderbilt, Phys. Rev. B 32, 8412 (1985)

${ }^{45}$ M. W. Schmidt et al., J. Comput. Chem. 14, 1347 (1993).

${ }^{46}$ C. Filippi and C. J. Umrigar, J. Chem. Phys. 105, 213 (1996); the Jastrow factor is further modified to deal with pseudoatoms and to completely separate the two body from the three-body terms.

${ }^{47}$ D. J. Clouthier and D. A. Ramsay, Annu. Rev. Phys. Chem. 34, 31 (1983); and references therein.

${ }^{48}$ M. B. Robin, Higher Excited States of Polyatomic Molecules (Academic, New York, 1985), Vol. 3, as cited in Ref. 5.

${ }^{49}$ A. Chutjan, J. Chem. Phys. 61, 4279 (1974).

${ }^{50}$ P. Jensen and P. R. Bunker, J. Mol. Spectrosc. 94, 114 (1982).

${ }^{51}$ S. Hirata and M. Head-Gordon, Chem. Phys. Lett. 314, 291 (1999).

${ }^{52}$ S. R. Gwaltney and R. J. Bartlett, Chem. Phys. Lett. 241, 26 (1995).

${ }^{53}$ M. R. Hachey, P. J. Bruna, and F. Grein, J. Phys. Chem. 99, 8050 (1995).

${ }^{54}$ P. Cronstrand, O. Christiansen, P. Norman, and H. Ågren, Phys. Chem. Chem. Phys. 2, 5357 (2000).

${ }^{55}$ M. von Arnim and S. D. Peyerimhoff, Chem. Phys. Lett. 210, 488 (1993).

${ }^{56}$ M. Dallos, T. Muller, H. Lischka, and R. Shepard, J. Chem. Phys. 114, 746 (2001).

${ }^{57}$ V. Bonačić-Koutecký and M. Persico, J. Am. Chem. Soc. 105, 3388 (1983).

${ }^{58}$ V. Bonačić-Koutecký, Theor. Chim. Acta. 68, 45 (1985). 